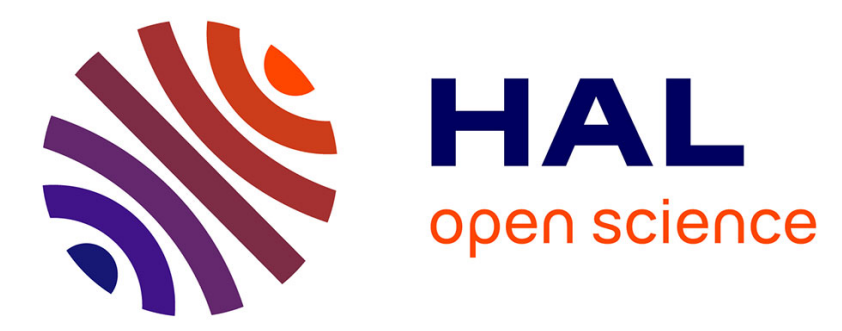

\title{
Nonlinear Model Predictive Control of the Air Path of a Turbocharged Gasoline Engine Using Laguerre Functions
} Jamil El Hadef, Sorin Olaru, Pedro Rodriguez-Ayerbe, Guillaume Colin, Yann Chamaillard, Vincent Talon

\section{- To cite this version:}

Jamil El Hadef, Sorin Olaru, Pedro Rodriguez-Ayerbe, Guillaume Colin, Yann Chamaillard, et al.. Nonlinear Model Predictive Control of the Air Path of a Turbocharged Gasoline Engine Using Laguerre Functions. 17th International Conference on System Theory, Control and Computing (ICSTCC), Oct 2013, Sinaia, Romania. 10.1109/ICSTCC.2013.6688959 . hal-00920494

\section{HAL Id: hal-00920494 \\ https://hal.science/hal-00920494}

Submitted on 18 Dec 2013

HAL is a multi-disciplinary open access archive for the deposit and dissemination of scientific research documents, whether they are published or not. The documents may come from teaching and research institutions in France or abroad, or from public or private research centers.
L'archive ouverte pluridisciplinaire HAL, est destinée au dépôt et à la diffusion de documents scientifiques de niveau recherche, publiés ou non, émanant des établissements d'enseignement et de recherche français ou étrangers, des laboratoires publics ou privés. 


\title{
Nonlinear Model Predictive Control of the Air Path of a Turbocharged Gasoline Engine Using Laguerre Functions
}

\author{
Jamil El Hadef, Sorin Olaru, Pedro Rodriguez-Ayerbe, Guillaume Colin, Yann Chamaillard, and \\ Vincent Talon
}

\begin{abstract}
Objectives in terms of pollutant emissions and fuel consumption reduction have led car manufacturers to enhance the technical definitions of combustion engines. The latter should now be considered as multiple-input multiple-output nonlinear systems with saturated actuators. This considerably increases the challenge regarding the development of optimal control laws under the constraints of constant cost reductions in the automotive industry.

In the present paper, the use of a nonlinear model predictive control (NMPC) scheme is studied for the air path control of a turbocharged gasoline engine. Specifically, a zero dimension physics-based model is combined with parameterization of the future control trajectory. The use of Laguerre polynomials is shown to increase flexibility for the future control trajectory at no cost in computational requirements. This increase in flexibility leads to an improvement of the transient response of the closed-loop with respect to traditional approaches. This practical application shows that this approach makes it easier to fine-tune the NMPC scheme when dealing with engine air path control.
\end{abstract}

\section{INTRODUCTION}

Current pollutant emission standards as well as fuel economy objectives have led car manufacturers to enhance the technical definitions of gasoline engines. In particular, the air path is constantly the seat of major evolutions with the addition of numerous new actuators: turbochargers, exhaust gas recirculation loops, variable valve timing, etc. As considered, gasoline engines have become multi-input multioutput nonlinear systems with saturated actuators. Over the years, model predictive control has proven to be a very good alternative for controlling nonlinear multivariable processes such as chemical plants [1]. However, until recently, calculation time considerations have prevented this type of approach from being extended to the automotive industry. Increasing computational capabilities combined with the socalled explicit model predictive control have overcome this limit and enlarged the range of possible applications [2-5], including automotive applications $[6,7]$

In discrete NMPC, one key element is modeling the future control trajectory, i.e. either the control signal $u$ or its increment $\Delta u$. In the case of rapid sampling, with respect to the control horizon, this may require a large number of

J. El Hadef is with the University of Orleans, Laboratoire PRISME, 8 rue Leonard de Vinci, 45000 Orleans, FRANCE and Renault SA, CTL, 1 allée de Cornuel, 91510 Lardy, FRANCE (e-mail: jamil.el-hadef@etu.univorleans.fr).

S. Olaru and P. Rodriguez-Ayerbe are with the E3S (Supelec System Science), Automatic Control Department, FRANCE (e-mail: sorin.olaru@supelec.fr).

G. Colin and Y. Chamaillard are with the University of Orleans.

V. Talon is with Renault SA (e-mail: vincent.talon@ renault.com). parameters to be identified, thus impacting on the feasibility of optimization and on computational requirements. In [8] the computational efficiency of the control problem is improved by adopting an approach based on wavelet decomposition. Alternatively in [6], the authors overcome this drawback by reducing the degrees of freedom of the control trajectory over the prediction horizon. The same issue is addressed in [9], where an expansion of the control trajectory based on Laguerre polynomials is applied to the linear case. This leads to a parsimonious description of a smooth control trajectory that minimizes the number of parameters to be identified. In this paper, this approach is extended to the nonlinear case and applied to the control of the air path of a turbocharged gasoline engine.

The paper is organized as follows. Section 2 presents insights into the physics-based model of the engine. Section 3 presents the general NMPC scheme as well as the parameterization strategy of the control trajectory. Simulation results are presented in section 4 . The conclusion summarizes the main outcome of the study and the next steps to be achieved.

\section{TURBOChARGED GASOLINE ENGINE}

The purpose of the study is to control the air path of a $1.2 \mathrm{~L}$ turbocharged gasoline engine as described in Fig.1.

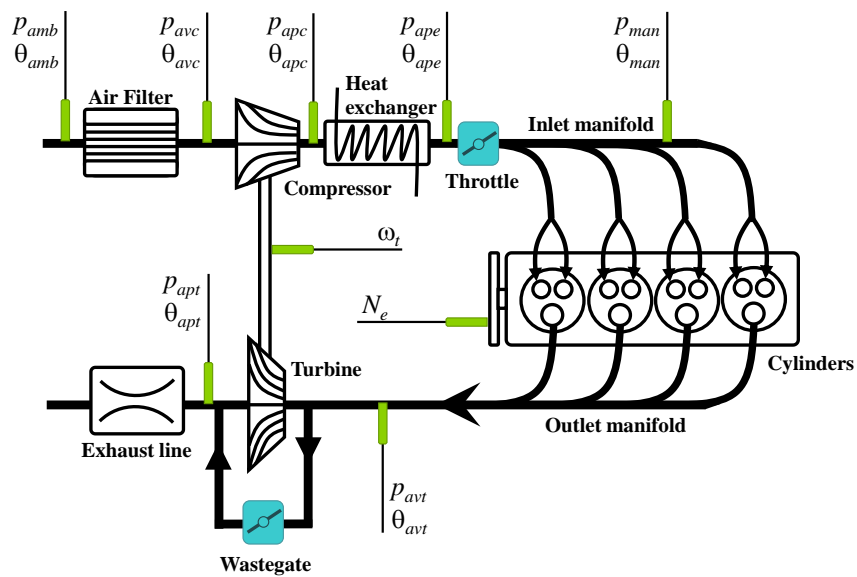

Figure 1. Overview of the gasoline engine used in this study ( $p$ stands for pressure, $\theta$ for temperature, $N_{e}$ and $\omega_{\mathrm{t}}$ are respectively the engine and turbocharger rotational speed). At the intake, the compressor and the heat exchanger successively increase the pressure and cool down the fresh air flow. Then, the throttle controls the air flow entering the inlet manifold. At the exhaust, the burnt air flow through the turbine is controlled by a wastegate. 
A nonlinear model predictive controller requires obtaining an analytic representation of the system [1, 3, 10]. In classical NMPC approaches, where the optimization problem is solved online, the computational time required to evaluate the model outputs is crucial. For this reason, authors usually rely on linearized models for describing internal combustion engines [11]. On the other hand, when considering explicittype approaches, the model is only used offline and using nonlinear modeling approaches then becomes possible. The combination of the zero-dimension and the mean value engine modeling approaches has proven to be a good compromise between accuracy, calibration effort and computational requirements. Moreover, in [7], it is demonstrated that this approach is suitable for the synthesis of an explicit control law.

\section{A. Mean Value Engine Model}

In this study, the engine air path is discretized following the zero-dimension philosophy. Each component on the air path is either defined as a reservoir or as a flow source. Hence, the model appears as a simple succession of flow restrictions and control volume sub-models (Fig. 2). The pressures in the latter correspond to model states and are governed by a set of differential equations. In order to keep the number of states of the model under control, temperature dynamics are neglected [12]: in each control volume, they are computed using algebraic relations that are not detailed below. Further information is provided in $[13,14]$ :

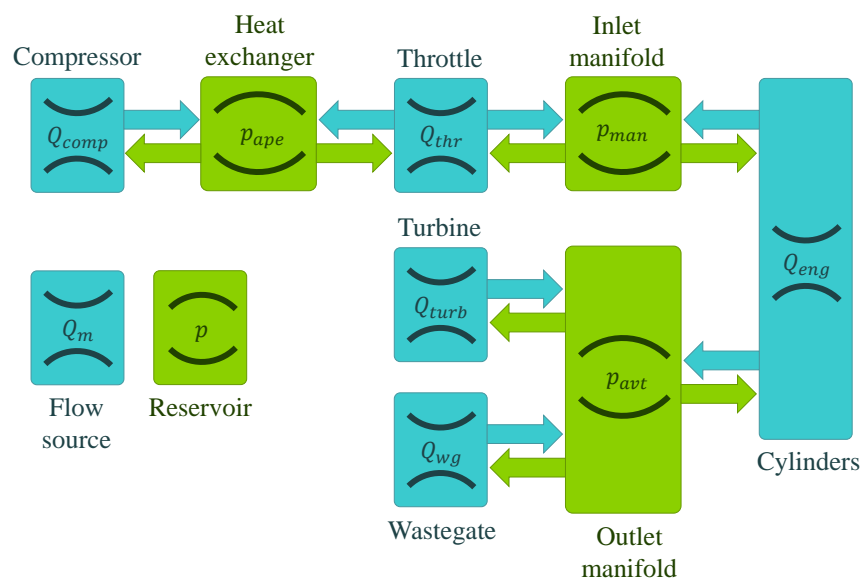

Figure 2. Overview of the engine model: each control volume is followed by a flow source, itself followed by another control volume and so forth. Each control volume represents a state of the system, i.e. in this particular case the model contains at least 3 states: $p_{\text {ape }}, p_{\text {man }}$ and $p_{\text {avt }}$.

\section{B. Reservoir model}

Euler's mass, energy and momentum equations are applied in each control volume in order to determine the pressure dynamic in the corresponding reservoir [14]:

$$
\dot{p}=\frac{\gamma r}{V}\left(Q_{m_{\text {in }}} \theta_{\text {in }}-Q_{m_{\text {out }}} \theta_{\text {out }}\right)
$$

where $\gamma$ is the ratio of specific heats, $r$ is the specific gas constant, $V$ is the volume of the reservoir, $\theta$ is the flow temperature and $Q_{m}$ is the mass flow rate. Indices in and out respectively stand for inlet and outlet.

This relationship can be applied to compute the dynamic of three model states:

$$
\left\{\begin{array}{l}
\dot{p}_{\text {ape }}=\frac{\gamma r}{V_{\text {ape }}}\left(Q_{\text {comp }} \theta_{\text {apc }}-Q_{t h r} \theta_{\text {ape }}\right) \\
\dot{p}_{\text {man }}=\frac{\gamma r}{V_{\text {man }}} \theta_{\text {man }}\left(Q_{t h r}-Q_{\text {eng }}\right) \\
\dot{p}_{\text {avt }}=\frac{\gamma r}{V_{\text {avt }}} \theta_{\text {avt }}\left(Q_{\text {eng }}+Q_{\text {fuel }}-Q_{t u r b}-Q_{w g}\right)
\end{array}\right.
$$

where $V_{\text {ape }}, V_{\text {man }}$ and $V_{\text {avt }}$ respectively represent the volume between the compressor and the throttle (including the heat exchanger), the volume of the intake manifold and the exhaust manifold volume (Fig. 1 and Fig. 2).

\section{Actuator orifice models}

The throttle and the wastegate are actuators that act as pure flow restrictions and respectively deliver $Q_{t h r}$ and $Q_{w g}$ in (2). Both flows can be computed using upstream and downstream pressures [15]:

$$
\left\{\begin{array}{l}
Q(u)=\frac{p_{u s}}{\sqrt{r T_{u s}}} A_{e f f}(u) \Pi^{\frac{1}{\gamma}} \sqrt{\frac{2 \gamma}{\gamma-1}\left(1-\Pi^{\frac{\gamma-1}{\gamma}}\right)} \\
\Pi=\max \left(\frac{p_{d s}}{p_{u s}},\left(\frac{2}{\gamma+1}\right)^{\frac{\gamma}{\gamma-1}}\right)
\end{array}\right.
$$

where $A_{\text {eff }}$ is the effective area of the orifice which depends on the actuator position $u$. The indices " $u s$ " and " $d s$ " respectively stand for upstream and downstream.

\section{Cylinder flow rate}

Let's denote $Q_{\text {eng }}$ the fresh air entering the cylinders. Assuming that the pressure in the inlet manifold is uniform, it is given by the speed-density equation [15]:

$$
Q_{\text {eng }}=\eta_{\text {vol }} \frac{p_{\text {man }} V_{c y l} N_{e}}{120 r \theta_{\text {man }}}
$$

where $p_{\text {man }}$ and $\theta_{\text {man }}$ are the manifold pressure and temperature, $V_{c y l}$ is the engine displacement, $N_{e}$ is the engine rotational speed and $\eta_{v o l}$ is the volumetric efficiency. The latter describes the ability of air to suck up air from the inlet manifold. A second order polynomial $\varphi$, calibrated on steady state test bench measurements, can be used to represent it:

$$
\eta_{\text {vol }}=\varphi\left(N_{e}, \frac{p_{\text {man }}}{\theta_{\operatorname{man}}}\right)
$$

\section{E. Compressor and turbine flow models}

Finally compressor and turbine flow rates, namely the last flow rates required to obtain $\dot{p}_{a p e}, \dot{p}_{\text {man }}$ and $\dot{p}_{a v t}$ from (12), can be computed by means of manufacturer look up tables:

$$
\left\{\begin{array}{l}
Q_{\text {comp }}=\Phi_{\text {comp }}\left(\pi_{\text {comp }}, \omega_{t}\right) \\
Q_{\text {turb }}=\Phi_{\text {turb }}\left(\pi_{\text {turb }}, \omega_{t}\right)
\end{array}\right.
$$

where $Q_{\text {comp }}$ and $Q_{\text {turb }}$ are respectively the compressor and turbine flow rates. $\Phi_{\text {comp }}$ and $\Phi_{\text {turb }}$ are nonlinear datamaps. Their inputs are the turbocharger rotational speed $\omega_{t}$ and the pressure ratios across the components, respectively $\pi_{\text {comp }}$ and $\pi_{t u r b}$.

At this stage, all the pressures in the model can be computed using (2). However, the dynamic of the engine cannot be fully described without taking into account the inertia of the turbocharger. In order to do this, a fourth state equation is added to the model in order to describe the dynamic of $\omega_{t}$ : 


$$
\dot{\omega}_{t}=\frac{1}{I}\left(\Gamma_{t u r b}-\Gamma_{c o m p}\right)
$$

where $I$ is the turbocharger inertia. $\Gamma_{\text {turb }}$ and $\Gamma_{\text {comp }}$ are respectively the turbine and compressor torques computed using their respective flow rates, inlet and outlet temperatures and $\omega_{t}$.

\section{CONTROLLER DESIGN}

\section{A. Nonlinear Model Predictive Control Tracking Scheme}

An iterative finite-time open loop nonlinear optimization problem is solved to compute the vector of optimal future actuators' positions with respect to a given objective function S. At each time step, only the first control move is applied to the real process before a new open-loop optimal problem is solved following the receding horizon principle $[1,3,10]$.

Let $\Delta \mathrm{u}$ be the vector of the difference of future control. S depends on $\Delta \mathrm{u}$ and any appropriate combination of the system states $\mathrm{x}$, the system outputs $\mathrm{y}$ and a vector of exogenous inputs $\sigma$. The latter contains, at least, the set points to be tracked. Then, given the current vectors of system states $\mathrm{x}_{0}$, previous controls $u_{0}$ and $\sigma$ at time instant $\mathrm{k}$, the discretized NMPC problem that is addressed is given by:

subject to

$$
S\left(\Delta u^{*}\right)=\min _{\Delta u(.)} \sum_{i=k}^{k+N_{p}} J(x(i), y(i), \sigma)
$$

$$
\begin{gathered}
x(k+1)=f(x(k), u(k), \sigma) \\
y(k)=g(x(k), u(k), \sigma) \\
u(k)=u(k-1)+\Delta u(k) \\
\underline{x} \leq x(k) \leq \bar{x} \\
\underline{u} \leq u(k) \leq \bar{u} \\
x(k)=x_{0} \\
u(k-1)=u_{0}
\end{gathered}
$$

where $H_{p}=N_{p} T_{s}$ is the prediction horizon and $T_{s}=10 \mathrm{~ms}$ is the control sampling interval. Together, $f$ and $g$ represent a nonlinear model of the system. Finally, $\underline{x}, \bar{x}, \underline{u}$ and $\bar{u}$ respectively stand for lower and upper bounds on the states and the control variables.

\section{B. Engine Model for NMPC}

In order to fit into the NMPC formulation above, the engine air path control, based on the model described in section 2, should be considered as follows:

$$
\begin{gathered}
x:=\left(p_{\text {ape }}, p_{\text {man }}, p_{\text {avt }}, \omega_{t}\right)^{T} \\
y:=\left(p_{\text {man }}, p_{\text {avt }}\right) \\
u:=\left(u_{\text {ap }}, u_{w g}\right) \\
\sigma=\left(p_{\text {man }}^{S P}, N_{e}, p_{a m b}, \theta_{\text {amb }}\right)
\end{gathered}
$$

where $f$ is given by (2) and (7) which describe the dynamic of the four states of the model (16).

For control design purposes, Euler's backward differentiation method is used to discretize (2) and (7) of the engine model. A minimum sampling time of 1 millisecond is required to ensure model convergence.

In this particular problem, no explicit state constraints, such as (12), are required. However, constraints on the control inputs are necessary in order to take into account physical saturation of the actuators.

$$
0 \leq\left(\begin{array}{l}
u_{\text {pap }} \\
u_{w g}
\end{array}\right) \leq 100 \%
$$

In order to track the inlet manifold pressure set point, while maximizing the engine efficiency, the thermodynamic cost function presented in [6] is used:

$$
J=\sum_{k=1}^{H_{p}} \alpha\left(p_{\text {man }}^{S P}-p_{\text {man }}\right)^{2}+\beta\left(\frac{p_{\text {avt }}}{p_{\text {man }}}\right)
$$

where $\alpha$ and $\beta$ are used to scale and penalize each term of the cost function.

\section{Parameterization of the control signal trajectory}

In this study, a set of orthonormal functions $l_{1}, l_{2}, \ldots, l_{N}$ is used to parameterize the trajectory of the difference of future control $\Delta u$. At any given instant $\mathrm{k}$, the expansion below is used for each actuator control trajectory:

$$
\Delta u(k)=\sum_{m=1}^{N} C_{m} l_{m}(k)
$$

where $N$ is the number of terms $l_{m}$ used to describe the control trajectory: as $N$ increases, the degrees of freedom of the control trajectory increase. $C_{m}=\left[\begin{array}{llll}C_{1} & C_{2} & \ldots & C_{N}\end{array}\right]^{T}$ is the vector of parameters to be identified. The problem of finding the optimal control signal over a given prediction horizon is then converted into finding the vector of weights $C_{m}$

\section{1) Laguerre polynomials}

In this study the set of Laguerre polynomials $L(k)=$ $\left[\begin{array}{llll}l_{1}(k) & l_{2}(k) & \cdots & l_{N}(k)\end{array}\right]^{T}$ was chosen to describe the control trajectory [9]. These are defined such that the $\mathrm{z}$ transfer function $\Gamma_{m}$ of the m-th Laguerre function $l_{m}$ is given by [16]:

$$
\Gamma_{m}(z)=\frac{\sqrt{\left(1-a^{2}\right)}}{z-a}\left[\frac{1-a z}{z-a}\right]^{m-1}
$$

where $a$ is called scaling factor and falls within $[0 ; 1[$.

In the time domain, the set of discrete Laguerre functions satisfies a difference equation [9]:

$$
L(k+1)=\Omega L(k)
$$

where $b=1-a^{2}$ in

$$
\Omega=\left[\begin{array}{ccccc}
a & 0 & 0 & \ldots & 0 \\
b & a & 0 & \ldots & 0 \\
-a b & b & a & \ldots & 0 \\
a^{2} b & -a b & b & \ldots & 0 \\
\vdots & \vdots & & & 0 \\
(-1)^{N-2} a^{N-2} b & (-1)^{N-3} a^{N-3} b & \ldots & b & a
\end{array}\right]
$$

The initial condition is given by:

$$
L(0)=\sqrt{b}\left[\begin{array}{lllll}
1 & -a & a^{2} & \ldots & (-1)^{N-1} a^{N-1}
\end{array}\right]^{T}
$$

When $a=0, L(k)$ becomes a set of pulses. In this case, the use of Laguerre polynomials for the parameterization of the control signal is equivalent to the traditional approach in NMPC design [9]. Conversely, for non-zero values, as a increases, the control horizon increases in the sense of temporal spectrum (Fig. 3). 

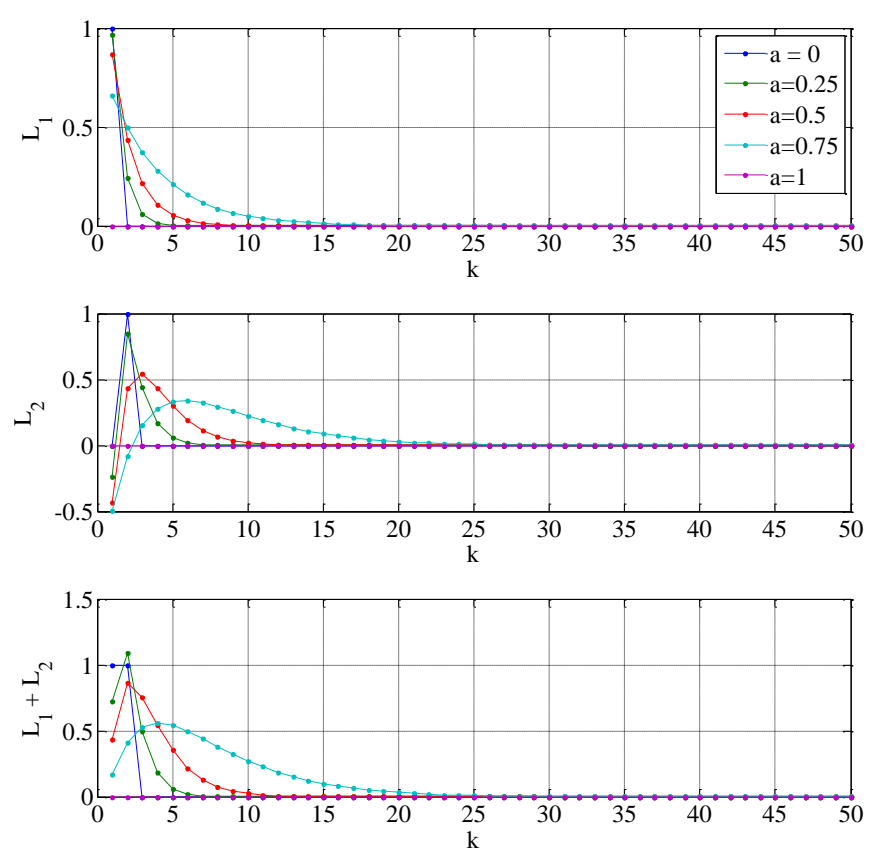

Figure 3. The first two levels of expansion of the Laguerre polynomials are plotted for various values of the scaling factor ( $a=0, a=0.25, a=0.5$, $a=0.75$ and $a=1$ ). On the bottom graph, the control trajectory is plotted for $C_{1}=C_{2}=1$. As the value of the scaling factor increases, the control horizon increases and the trajectory becomes less aggressive. When $a=0$, it is evident that the Laguerre polynomials become a set of pulses, respectively at $k=1$ and $k=2$.

\section{2) Computing the control increment}

Given the vector of parameters $\left[\begin{array}{llll}C_{1} & C_{2} & \ldots & C_{N}\end{array}\right]^{T}$, the sequence of control increments over the prediction horizon $\mathrm{N}_{\mathrm{p}}$ is given by the matrix equation:

$$
\left(\begin{array}{c}
\Delta u(k) \\
\Delta u(k+1) \\
\vdots \\
\Delta u\left(k+N_{p}\right)
\end{array}\right)=\mathbb{L}\left(\begin{array}{c}
C_{1} \\
C_{2} \\
\vdots \\
C_{N}
\end{array}\right)
$$

where

$$
\mathbb{L}=\left(\begin{array}{cccc}
l_{1}(k) & l_{2}(k) & \cdots & l_{N}(k) \\
l_{1}(k+1) & l_{2}(k+1) & \cdots & l_{N}(k+1) \\
\vdots & \vdots & & \vdots \\
l_{1}\left(k+N_{p}\right) & l_{2}\left(k+N_{p}\right) & \cdots & l_{N}\left(k+N_{p}\right)
\end{array}\right)
$$

3) Constraints on the control trajectory

In this particular problem, no explicit state constraints, such as (12), are required. However, constraints on the control inputs are required to take physical saturation of the actuators into account:

$$
\left(\begin{array}{l}
\frac{u_{p a p}}{u_{w g}}
\end{array}\right) \leq\left(\begin{array}{l}
u_{p a p} \\
u_{w g}
\end{array}\right) \leq\left(\overline{\overline{u_{p a p}}}\right)
$$

From (11) and (27), the sequence of control signals is given by:

$$
\left(\begin{array}{c}
u(k) \\
u(k+1) \\
\vdots \\
u\left(k+N_{p}\right)
\end{array}\right)=\mathbb{L}_{\Sigma}\left(\begin{array}{c}
C_{1} \\
C_{2} \\
\vdots \\
C_{N}
\end{array}\right)+u(k-1)
$$

where

$$
\mathbb{L}_{\Sigma}=\left(\begin{array}{cccc}
l_{1}(k) & l_{2}(k) & \cdots & l_{N}(k) \\
\sum_{i=1}^{2} l_{1}(k+i) & \sum_{i=1}^{2} l_{2}(k+i) & \cdots & \sum_{i=1}^{2} l_{N}(k+i) \\
\vdots & \vdots & & \vdots \\
\sum_{i=1}^{N_{p}} l_{1}(k+i) & \sum_{i=1}^{N_{p}} l_{2}(k+i) & \cdots & \sum_{i=1}^{N_{p}} l_{N}(k+i)
\end{array}\right)(31)
$$

The constraints on the amplitude of the inputs are then given by:

$$
\left(\begin{array}{cc}
\mathbb{L}_{\Sigma} & 0_{r} \\
-\mathbb{L}_{\Sigma} & 0_{r} \\
0_{r} & \mathbb{L}_{\Sigma} \\
0_{r} & -\mathbb{L}_{\Sigma}
\end{array}\right)\left(\begin{array}{c}
C_{1} \\
C_{2} \\
\vdots \\
C_{N} \\
C_{1} \\
C_{2} \\
\vdots \\
C_{N}
\end{array}\right)<\left(\begin{array}{l}
\llbracket \overline{u_{p a p}}-u_{p a p}(k-1) \rrbracket \\
\llbracket u_{p a p}(k-1)-u_{p a p} \rrbracket \\
\llbracket \overline{u_{w g}}-u_{w g}(k-1) \rrbracket \\
\llbracket u_{w g}(k-1)-u_{w g} \rrbracket
\end{array}\right)
$$

where $0_{\mathrm{r}}$ is a zero matrix of appropriate dimensionality. In the same manner, $\llbracket . . . \rrbracket$ denotes a vector of appropriate length.

\section{Simulation Results}

\section{A. Calibration of the control scheme}

1) Number of terms in the expansion

The first parameter of the control scheme is the dimension of the Laguerre polynomials set, i.e. $N$. As the number of terms increases, the flexibility of the trajectory increases [9]. However, the computational requirements to solve the optimization problem also increase with $N$. Fig. 4 and Fig. 5 present the performances of such a controller for different values of $N$. The inlet manifold pressure set point $p_{\text {man }}^{S P}$ is moved from 0.6 bar (low load), respectively 0.9 bar, to 1.6 bar (high load) while the engine speed is kept constant at $2,000 \mathrm{rpm}$, respectively 5,000 rpm. The prediction horizon is chosen in agreement with the settling time of the system, i.e. $H_{p}=200 \mathrm{~ms}$. A scaling factor $a=0.5$ proved to be representative of the average controller performances.

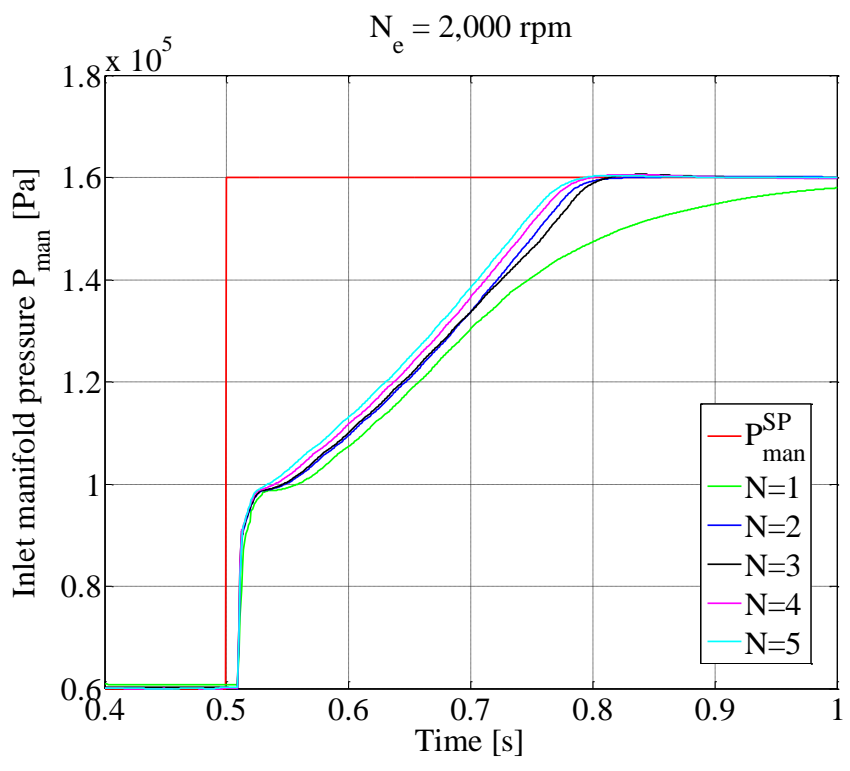

Figure 4. Influence of the number of Laguerre polynomial terms used to describe the future control trajectory at $2,000 \mathrm{rpm}$. From $N=2$ to $N=5$ the controllers present similar performances. $H_{p}=200 \mathrm{~ms}$ and $a=0.5$. 


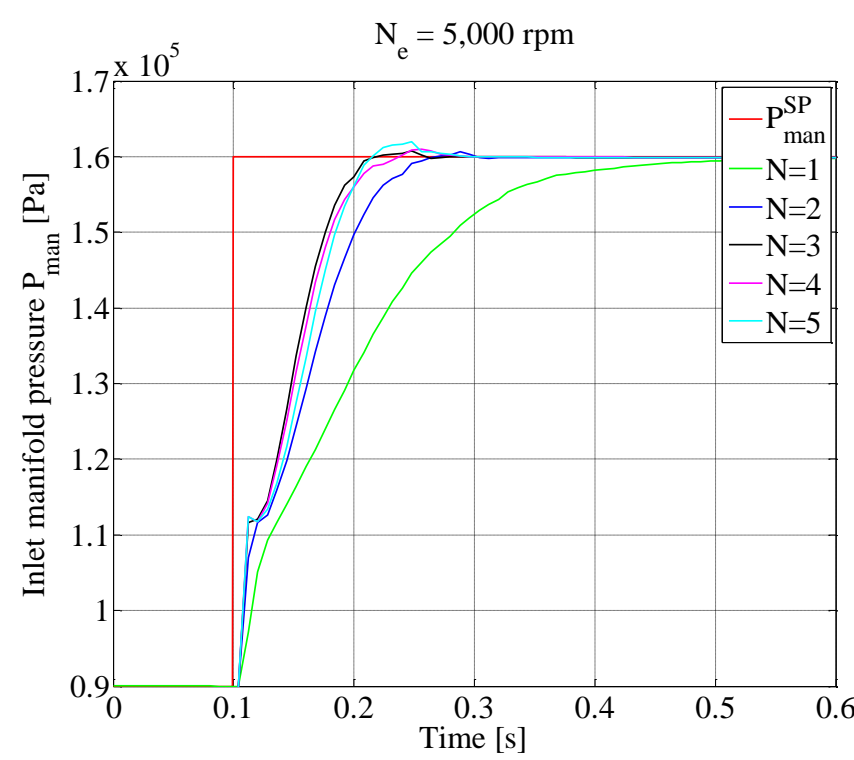

Figure 5. Influence of the number of Laguerre polynomial terms used to describe the future control trajectory at 5,000 rpm. Between $N=2$ and $N=5$ the controllers present similar performances. $H_{p}=200 \mathrm{~ms}$ and $a=0.5$.

Using only one Laguerre polynomial to build the control trajectory considerably reduces the closed-loop speed (Fig. 4 and Fig. 5). Then from $N=2$ to $N=5$ increasing the number of terms does not improve the control performances. Since the objective is to minimize the computational requirements, a 2-level expansion was chosen for the rest of the study. This corresponds to determining two parameters for each actuator at each sampling time. This problem was solved using the trust-region reflective algorithm method implemented in Matlab®. The algorithm converges within 20 iterations when initialized with $\Delta u=0$ over the prediction horizon.

\section{2) Prediction horizon}

As suggested above, the prediction horizon of the system is usually selected in agreement with the settling time of the system. In the case of a turbocharged gasoline engine, it can vary from about $100 \mathrm{~ms}$ to $300 \mathrm{~ms}$. In Fig. 6 and Fig. 7, the performances of the Laguerre polynomial based NMPC scheme are depicted for different prediction horizons: 100, 200, 300 and $500 \mathrm{~ms}$. Two engine rotational speeds : 2,000 rpm and 5,000 rpm, are presented. The same inlet manifold pressure set point step as before is used. The scaling factor is kept at 0.5 .

The influence of the prediction horizon on the response of the closed-loop system depends on the engine rotation speed. In fact, at low rotational speeds, the settling time of the engine does not seem to be affected by the prediction horizon that is chosen (Fig. 6). However, for high rotational speeds, the settling time of the system depends nonlinearly on the prediction horizon. In particular, using a prediction horizon of $100 \mathrm{~ms}$ considerably accelerates the engine response. For a prediction horizon greater than $200 \mathrm{~ms}$, the influence rapidly decreases (Fig. 7).

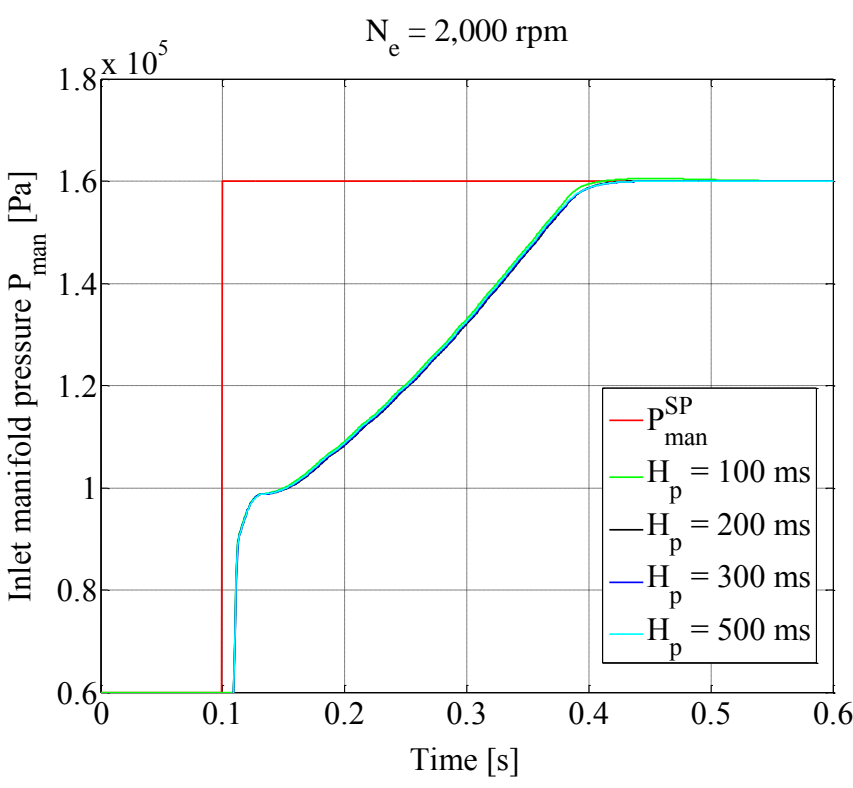

Figure 6. Influence of the prediction horizon $H_{p}$ on the response of the engine at 2,000 rpm. At this engine speed, the response of the system is independent of the prediction horizon. $N=2$ and $a=0.5$.

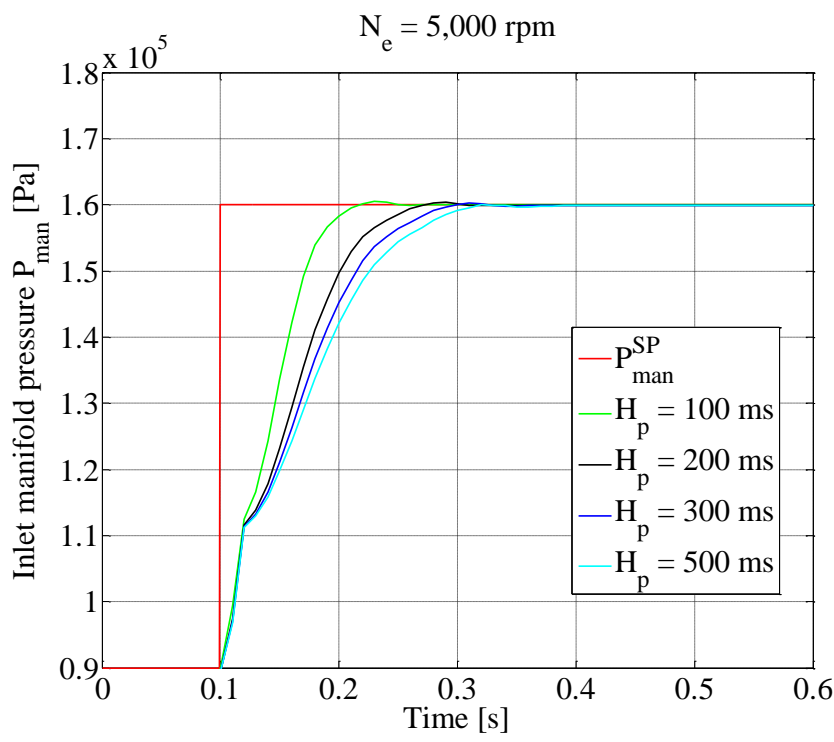

Figure 7. Influence of the prediction horizon $H_{p}$ on the response of the engine at 5,000 rpm.. It can be noticed that the settling time of the system depends nonlinearly on the prediction horizon. In particular, it varies by about $40 \%$ between $H_{p}=100 \mathrm{~ms}$ and $H_{p}=200 \mathrm{~ms}$ while varying by only

$4 \%$ between $H_{p}=200 \mathrm{~ms}$ and $H_{p}=300 \mathrm{~ms} . \mathrm{N}=2$ and $a=0.5$.

As such, a reasoned choice of the prediction horizon should take into account the dynamic of the system at a high rotational speed. Here $H_{p}=200 \mathrm{~ms}$ appears to be a good compromise between computation time and stability.

3) Scaling factor

The scaling factor of the Laguerre polynomials makes it possible to control the dynamic of the closed-loop system by modifying the control horizon $H_{c}$ [9] (Fig. 3). Fig. 8 and Fig. 9 present the closed loop response of the system to an inlet manifold pressure set point step for different values of the scaling factor. Two cases are presented: at 2,000 rpm and 
5,000 rpm. In accordance with the previous sub section, $N=2$ and $H_{p}=200 \mathrm{~ms}$.

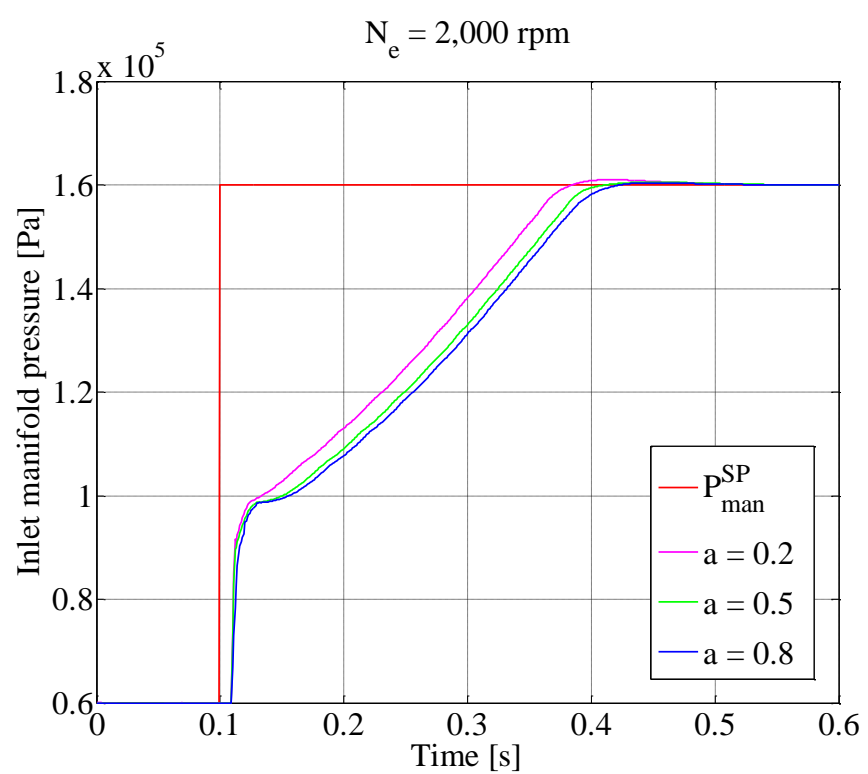

Figure 8. Influence of the scaling factor $a$ on the response of the engine at $2,000 \mathrm{rpm}$. It can be noticed that the scaling factor has a nonlinear effect on the closed-loop response. However at this rotational speed, the effect is not significant. $N=2$ and $H_{p}=200 \mathrm{~ms}$.

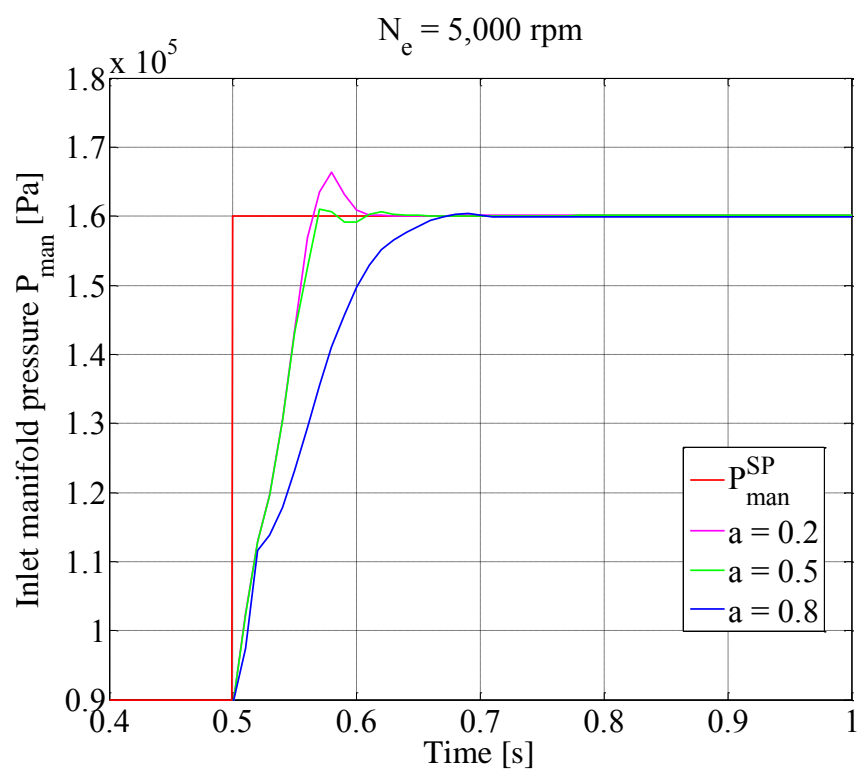

Figure 9. Influence of the scaling factor $a$ on the response of the engine at $5,000 \mathrm{rpm}$. The scaling factor significantly modifies the shape of the response in a nonlinear fashion. $N=2$ and $H_{p}=200 \mathrm{~ms}$.

At low rotational speeds, the influence of the scaling factor on the settling time of the system is small but still nonlinear: a bigger change is observed between $a=0.2$ and $a=0.5$ than between $a=0.5$ and $a=0.8$. At high rotational speeds, the same nonlinear effect is observed but the scaling factor has much more influence on the shape of the closed-loop response. In both cases, as expected in Fig. 3, the smaller the scaling factor is the faster the controller is.
Again a considered decision on the scaling factor must take into account the highest engine rotational speeds.

\section{B. Transient performances of the control scheme}

Based on the results presented before, the set of calibration parameters $N=2, H_{p}=200 \mathrm{~ms}$ and $a=0.8$ was selected. Fig. 10 depicts the transient performances of the nonlinear predictive controller on a representative part of a 65 -second realistic driving cycle. The engine speed varies from 1,000 rpm to 5,500 rpm while engine load varies from low to high. Actuator positions vary from fully closed to fully open (including sudden opening).

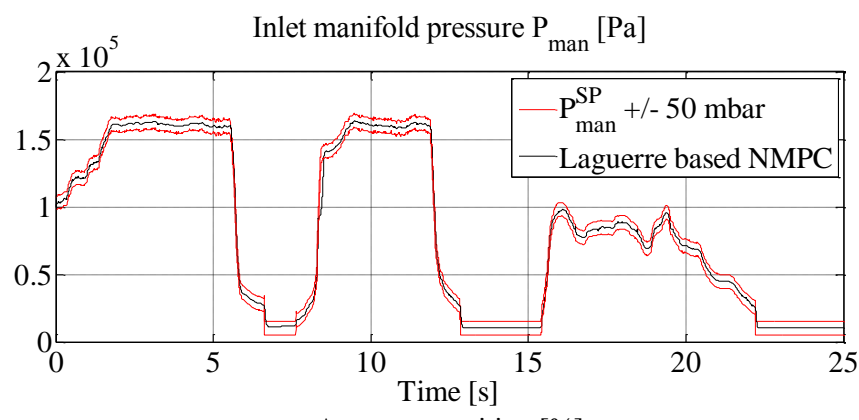

Actuators position $[\%]$

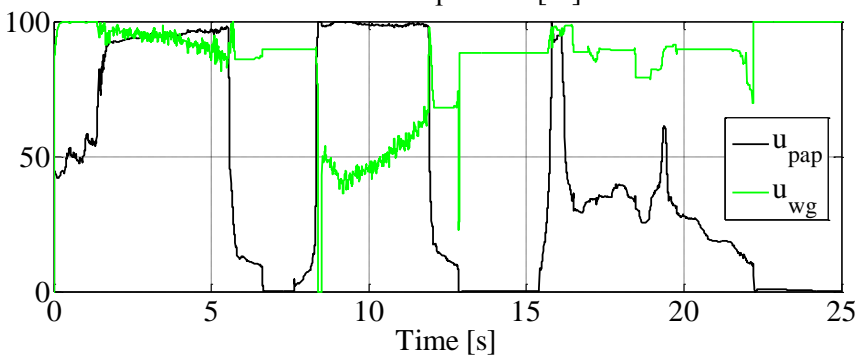

Figure 10. Inlet manifold pressure tracking on a realistic driving cycle (black line). The controller is parameterized as follows: $N=2, H_{p}=200 \mathrm{~ms}$ and $a=0.8$. The inlet manifold pressure reference trajectory is depicted through upper and lower 50 mbar tolerance intervals (red lines). The bottom plot displays the corresponding throttle (green line) and wastegate positions (black line).

The nonlinear model predictive controller presented in this paper leads to tracking performances in agreement with standard specifications in the automotive industry: the inlet manifold trajectory stays within the $+/-50$ mbar tolerance interval and has no static error (Fig. 4 to 8 ).

\section{Comparison with the traditional NMPC design}

The previous section showed which considerations need to be taken into account regarding the dynamic of the system and its dependence on the engine rotational speed. Altogether, the calibration effort that is required to tune the NMPC scheme based on Laguerre polynomials is quite similar to the one required to calibrate a traditional NMPC design. In fact, only three parameters, namely $N, H_{p}$ and $a$ need to be determined. However, when comparing the performances of the two control schemes, great differences emerge.

As detailed above, in the new approach, using $N=2$ requires solving a 4-dimension problem at each time step (2 parameters need to be determined for each actuator). This is 
similar to a standard NMPC scheme using a control horizon of 1. On Fig. 11 and 12, the performances of these two schemes are presented side by side. The prediction horizon is $200 \mathrm{~ms}$ in both cases and both problems are solved using a trust-region reflective method which converges within 20 iterations.

At low rotational speeds, the shape of the response is quite similar for both NMPC schemes. On Fig. 11, the controller based on Laguerre polynomials expansion leads to a smaller settling time without inducing any overshoot.

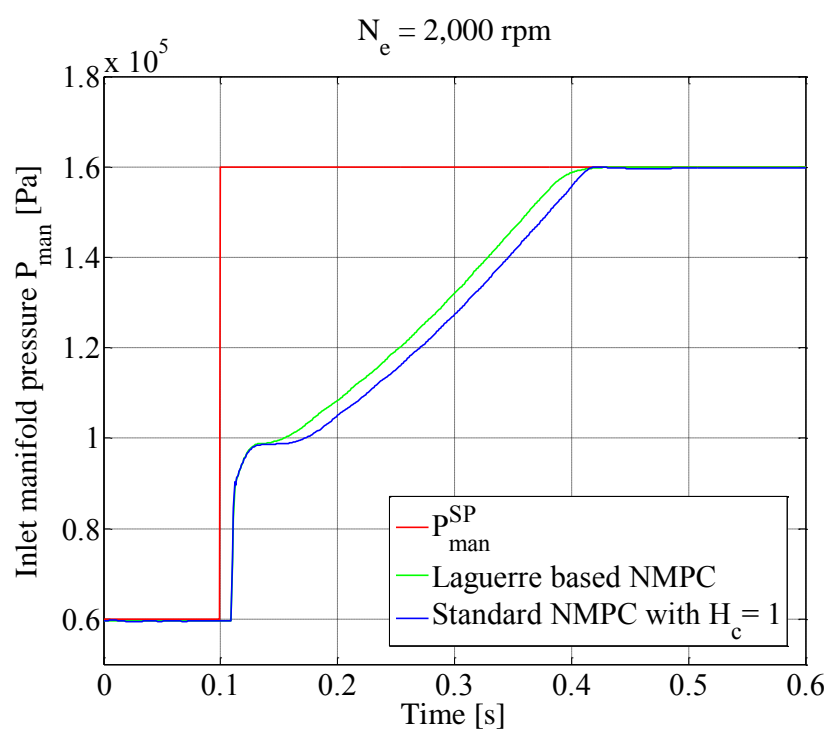

Figure 11. Comparison of the tracking performances at 2,000 rpm of a standard NMPC control scheme with $H_{c}=1$ (blue line) and the Laguerre polynomial based NMPC control scheme (green line). The parameterization of the control trajectory leads to better performances than the standard approach when considering $H_{c}=1$.

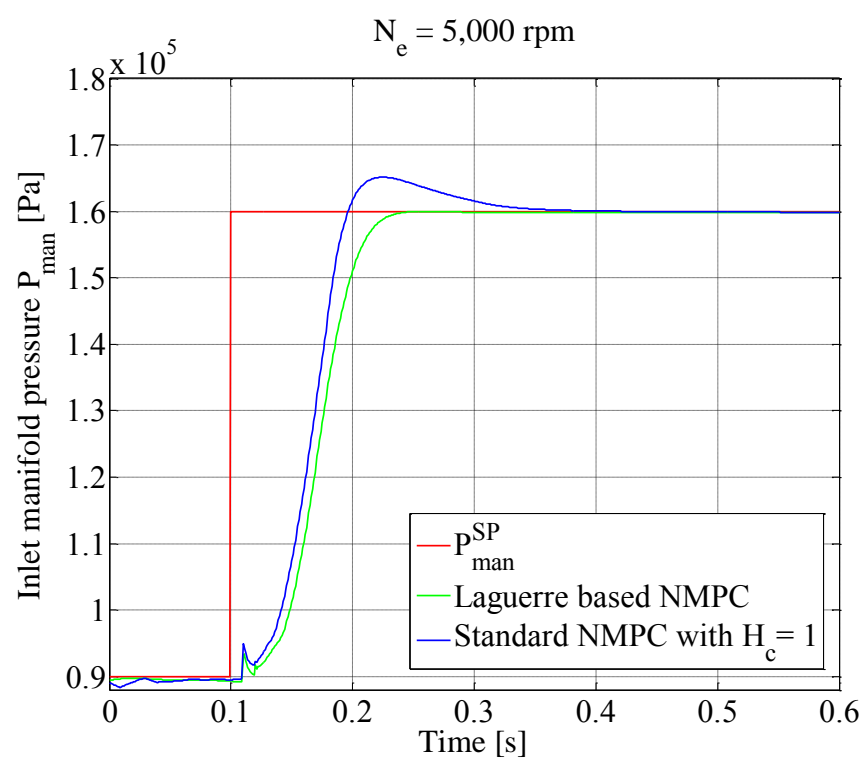

Figure 12. Comparison of the tracking performances at 5,000 rpm of a standard NMPC control scheme with $H_{c}=1$ (blue line) and the Laguerre polynomial based NMPC control scheme (green line). The parameterization of the control trajectory leads to better performances than the standard approach when considering $H_{c}=1$.
At high rotational speeds, the difference is much bigger. On Fig. 12, the controller based on the traditional approach presents an overshoot of 0.5 bar which is not acceptable with respect to automotive standards, whereas, the greater flexibility of the controller based on a parameterized control trajectory leads to a faster settling time with an insignificant overshoot.

This type of behavior is observed thoughout the entire engine operating range with more sensitivity at high loads and high rotational speeds. In fact, in these types of conditions, a satisfactory approximation of the future control law will usually require a large number of forward shift operators [9]. In those conditions, the Laguerre polynomials based parameterization has been shown to improve the performances of the closed-loop when considering engine air path control.

\section{CONCLUSION}

In this paper, the control of the air path of a turbocharged gasoline engine is achieved using a NMPC scheme based on parameterizing the future control trajectory. This parameterization of the control trajectory is based on Laguerre polynomials and is very similar to the one presented in [9]. The main contribution of this study is that it was put into practice on a nonlinear practical application and combined with a fully physics-based nonlinear engine model.

In this approach, the problem of finding the optimal future control trajectory is converted into determining a set of multiplying coefficients for Laguerre polynomials. This leads to a parsimonious description of the control trajectory that improves the response of the system in terms of speed and stability. Moreover, both the computational requirements and the calibration efforts are maintained with respect to traditional approaches.

Further extensions will go in the direction of implementing the controller in real-time using the explicitapproach proposed in [7].

\section{REFERENCES}

[1] E. F. Camacho and C. Bordons, Model Predictive Control, 2 ed London: Springer, 2004.

[2] A. Bemporad, et al., "The Explicit Solution of Model Predictive Control via Multiparametric Quadratic Programming," in American Control Conference, Chicago, 2000.

[3] L. Del Re, et al., Automotive Model Predictive Control. Berlin: Springer, 2010.

[4] S. Di Cairano, et al., "Model Predictive Control of Magnetically Actuated Mass Spring Dampers for Automotive Applications," Int. J. of Control, vol. 80, pp. 1701-1716, 2007.

[5] A. Grancharova and T. A. Johansen, Explicit Nonlinear Model Predictive Control. Berlin: Springer, 2012.

[6] J. El Hadef, et al., "Explicit-Ready Nonlinear Model Predictive Control of the Air Path of a Turbocharged Spark-Ignited Engine," in 7th IFAC Symposium on Advances in Automotive Control, Tokyo, Japan, 2013.

[7] J. El Hadef, et al., "Explicit Nonlinear Model Predictive Control of the Air Path of a Turbocharged Spark-Ignited Engine," presented at the IEEE Multi-Conference on Systems and Control, Hyderabad, India, 2013. 
[8] J. H. Lee, et al., "Improving Computational Efficiency of Model Predictive Control Algorithm Using Wavelet Transformation," International Journal of Control, vol. 61, pp. 859-883, 1995.

[9] L. Wang, "Discrete Model Predictive Controller Design Using Laguerre Functions," Journal of Process Control, vol. 14, pp. 131-142, 2004.

[10] H. J. Ferreau, et al., "Fast Nonlinear Model Predictive Control of Gasoline Engine," in International Conference on Control Applications, Munich, Germany, 2006.

[11] J. Pekar, et al., "Experimental Results for Sensor Selection and Multivariable Controller Design for a Heavy-Duty Diesel Engine," presented at the IFAC Workshop on Engine and Powertrain Control, Simulation and Modeling, France, 2012.

[12] E. Hendricks, "Isothermal versus Adiabatic Mean Value SI Engine Models," 3rd IFAC Workshop, Advances in Automotive Control, pp. 373-378, 2001.

[13] J. El Hadef, et al., "Turbocharged SI Engine Models for Control," in The 11th International Symposium on Advanced Vehicle Control AVEC'12, Seoul, Korea, 2012.

[14] L. Eriksson, "Modeling and Control of Turbocharged SI and DI Engines," Oil \& Gas Science and Technology - Rev. IFP Energies nouvelles, vol. 62, pp. 523-538, 2007.

[15] J. B. Heywood, Internal Combustion Engines Fundamentals: McGrawHill, 1988.

[16] B. Wahlberg, "System Identification Using Laguerre Models," IEEE Transactions on Automatic Control, vol. 36, pp. 551-562, 1991. 